\title{
IDIOPATHIC PYODERMA GANGRENOSUM OR A SYSTEMIC DISEASE PREDICTOR?
}

\author{
Todor Yordanov ${ }^{1}$, Jenya Dimitrova ${ }^{2}$, Ivanka Temelkova ${ }^{1}$, Tsveta Kalinova ${ }^{2}$, Neli Koleva ${ }^{3}$, \\ Sonya Marina ${ }^{1}$ \\ ${ }^{1}$ Department of Skin and Venereal Diseases, \\ Medical Institute of the Ministry of Internal Affairs \\ ${ }^{2}$ Department of Infectious Diseases, Parasitology and Dermatovenereology, \\ Faculty of Medicine, Medical University of Varna \\ ${ }^{3}$ Department of Clinical Pathology, Medical Institute of the Ministry of Internal Affairs
}

\begin{abstract}
Pyoderma gangrenosum (PG) is an auto inflammatory non-infectious neutrophilic dermatosis often presenting with pustules or nodules that progress to ulcers. PG has specific clinical features and noncharacteristic histology findings. Some systemic diseases such as Crohn's disease, ulcerative colitis, rheumatoid arthritis, hematologic malignancy or autoimmune disease are commonly associated with PG. One of the most characteristic features of pyoderma gangrenosum is pathergy, which is the appearance of new lesions at sites of trauma, including surgical wounds.
\end{abstract}

We present a 62-year-old male with a large ulceration on the left lower leg after a bee sting.

Keywords: pyoderma gangrenosum, neutrophilic dermatosis, ulcers, pathergy

\section{INTRODUCTION}

Pyoderma gangrenosum (PG) is a rare inflammatory neutrophilic dermatosis of unknown etiology. It may be associated with a coexisting systemic disease, most commonly Crohn's disease, ulcerative colitis, rheumatoid arthritis, hematologic malignancy or autoimmune disease $(1,2)$. In most of the cases the initial lesion is a papule, pustule, or nodule appearing after minor trauma, that progresses

Address for correspondence:

Jenya Dimitrova

Faculty of Medicine

Medical University of Varna

55 Marin Drinov St

9002 Varna

e-mail:dimitrova_derm@abv.bg

Received: August 262020

Accepted: September 9, 2020 to painful, slowly growing ulcer. It is often misdiagnosed as a soft tissue infection that can coincidentally improve due to the systemic antibiotic therapy and wound care $(1,2,3)$.

We present a case of pyoderma gangrenosum persisting for 7 months, not associated with systemic disease.

\section{CASE REPORT}

A 62-year-old male presented to a dermatology clinic in 2019 with a history of bee sting after which a non-healing painful ulcer developed on the anteromedial aspect of the left shin. The lesion was large and deep, with a diameter of $15 / 20 \mathrm{~cm}$, elevated borders and erythematous periphery (Fig. 1, Fig. 2).

Previously, the patient was treated with local antiseptic, proteolytic enzyme and a systemic antibiotic with unsatisfactory improvement. 


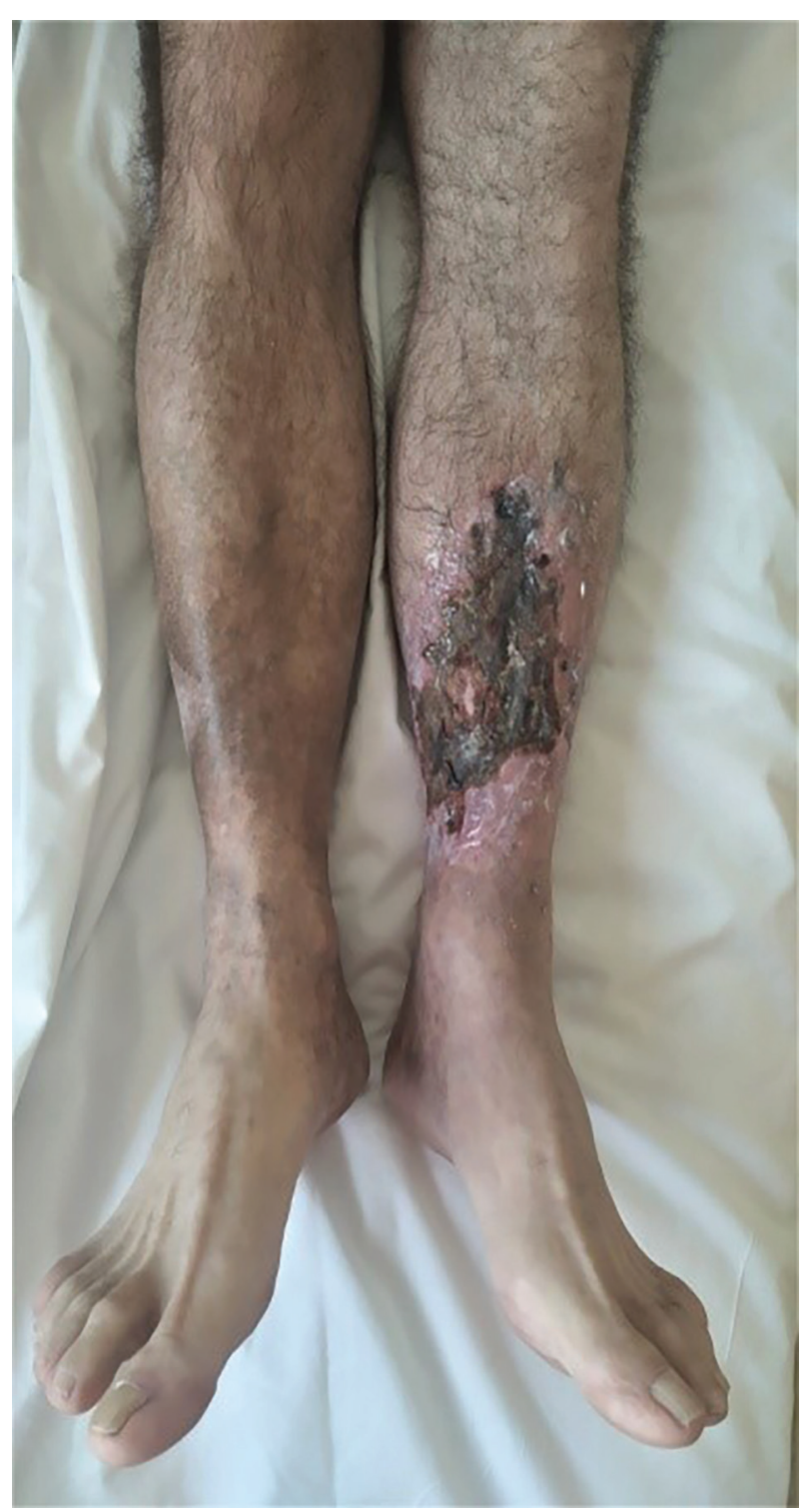

Fig. 1. Initial clinical findings

The laboratory tests showed: erythrocytes $(\mathrm{RBC})-3.0310^{\wedge} 12 / \mathrm{l}$; erythroblasts - 10^9/L; hemoglobin (HGB) - 118.0 g/L; hematocrit (HCT) -0.326 1/l ; MCV - 107.7 fl; MCH - 39.0 pg; MCHC - 362.0 g/L; granulocytes (Gran) \% - 77.6\%; lymphocytes (Lym) \% - 15.0\%; iron binding capacity (IBC) - 40.3 $\mu \mathrm{mol} / \mathrm{L}$; gamma-glutamyl transferase (GGT) -94.0 $\mathrm{U} / \mathrm{L}$; procalcitonin $-0.4249 \mathrm{ng} / \mathrm{mL}$; hsCRP -70.4 $\mathrm{mg} / \mathrm{L}$; ferritin - $365.58 \mathrm{ng} / \mathrm{mL}$; bilirubin total - 12.9 $\mu \mathrm{mol} / \mathrm{L}$; bilirubin direct $-4.8 \mu \mathrm{mol} / \mathrm{L}$. Urine tests: blood ++; ketone bodies (+); urobilinogen ++ ; bilirubin +++ ; leucocytes - neg. Microbiology culture test

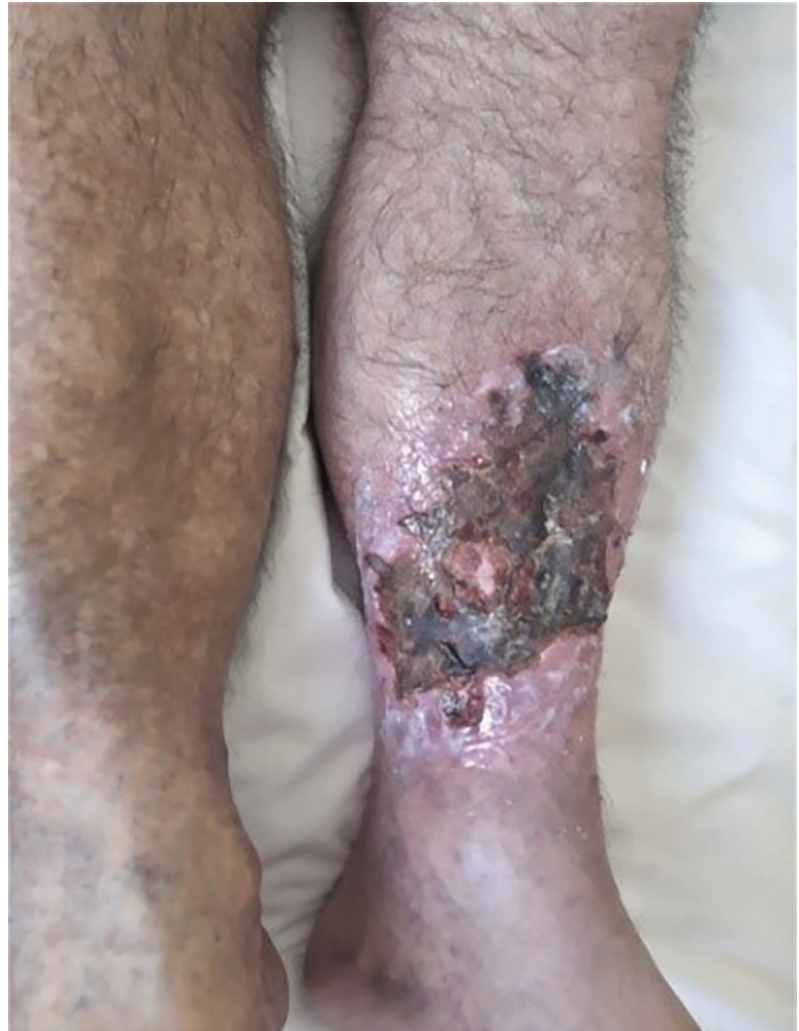

Fig. 2. Initial clinical findings (closer look)

of a sample taken from the ulcer found Pseudomonas aeruginosa.

Doppler ultrasound of the leg, chest X-ray, gastro-, colonoscopy and abdominal ultrasound of the patient found no significant abnormalities.

A hematologist and a gastroenterologist were consulted and they recommended treatment with folic acid, vit. B6, vit. B12 and ursodeoxycholic acid.

Skin biopsy from the border of the lesion was performed. The histologic findings are: pseudoepitheliomatous hyperplasia with intraepidermal neutrophilic abscesses and spongiosis of the epidermis. In the dermis diffuse inflammatory infiltrate containing numerous neutrophils was observed (Fig. 3, Fig. 4, Fig. 5).

The clinical course with pathergic phenomenon, unresponsiveness to antibiotic therapy and histopathologic features of sterile neutrophilic infiltration led to diagnosis pyoderma gangrenosum.

Systemic therapy with ciprofloxacin $200 \mathrm{mg}$ twice daily, 6-methylprednisolone $40 \mathrm{mg}$ i.v., diaminodiphenyl sulfone (DDS) $50 \mathrm{mg}$ twice daily and 
Todor Yordanov, Jenya Dimitrova, Ivanka Temelkova et al.

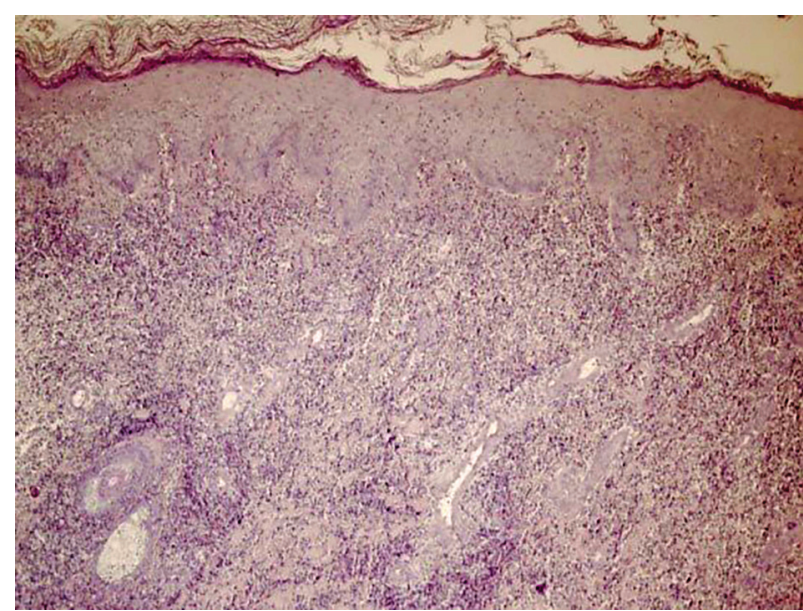

Fig. 3. Histologic findings, magnification $x 40$

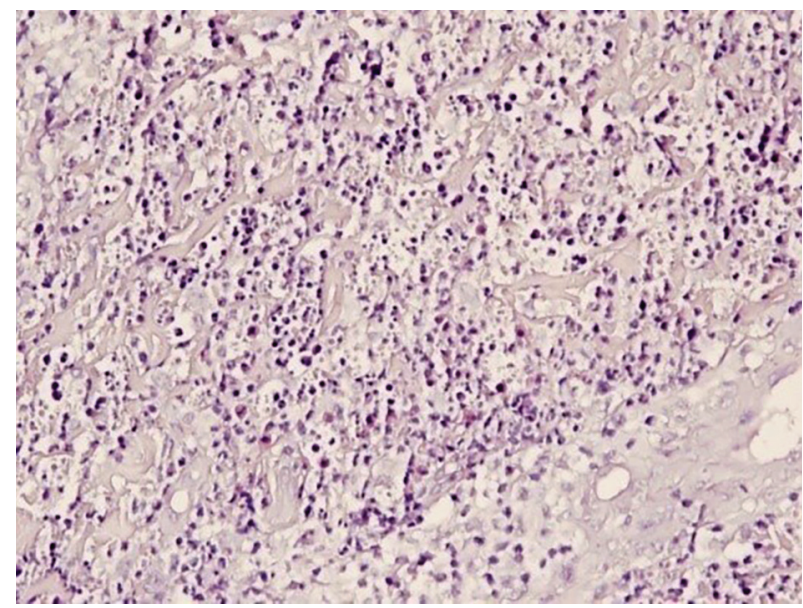

Fig. 4. Histologic findings, magnification $\times 100$

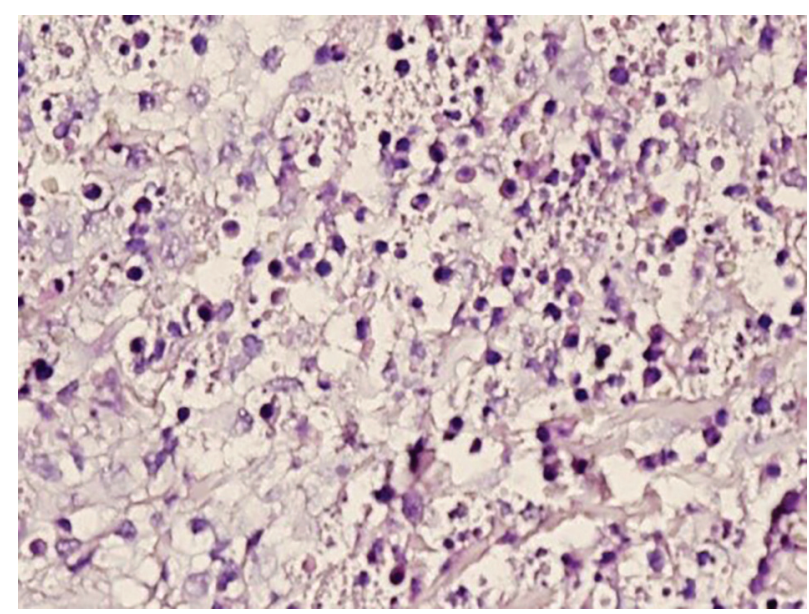

Fig. 5. Histologic findings, magnification $\times 200$

esomeprazole was initiated. Locally, $\mathrm{KMnO} 4$ compresses were applied.
Clinical improvement was observed in the first 10 days of the therapeutic regimen. Significant part of the devitalized tissue was removed. The size and the depth of the ulcer decreased. There were no signs of bacterial contamination (Fig. 6).

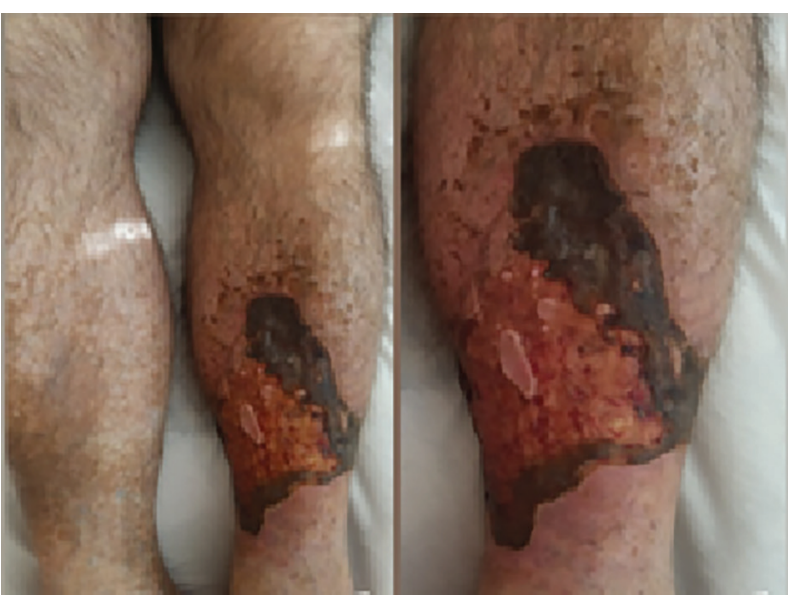

Fig. 6. Clinical improvement observed after 10 days of therapy

\section{DISCUSSION}

Pyoderma gangrenosum (PG) is a neutrophilic dermatosis, with characteristic clinical and noncharacteristic histology findings, first described by Brocq et al. in 1916 as "phagedenisme geometrique/ geometric phagedenism" $(2,3,4)$. Although the etiopathogenesis of PG is still unclear, there are increasing evidence that it shares common pathogenic mechanisms with other autoimmune disorders $(5,6,7)$. Neutrophilic dysfunction, systemic inflammation, and associated genetic factors are all involved in the formation of PG ulcers $(8,9)$.

Previously, there were no clinical criteria distinguishing PG from necrotizing soft tissue infections (10). Recently, E. Maverakis et al. suggested one major and 4 of 8 minor criteria that can be used to diagnose PG with high specificity (11). The major criterion is biopsy of an ulcer edge demonstrating neutrophilic infiltrate and the 8 minor criteria are: (1) exclusion of infection; (2) pathergy; (3) history of inflammatory bowel disease or inflammatory arthritis; (4) history of papule, pustule, or vesicle ulcerating within 4 days of appearing; (5) peripheral erythema, undermining border, and tenderness at ulceration site; (6) multiple ulcerations, at least 1 on an an- 
terior lower leg; (7) cribriform or "wrinkled paper" scar(s) at healed ulcer sites; and (8) decreased ulcer size within 1 month of initiating immunosuppressive medication(s) (11). Applying those criteria yields 86\% to $90 \%$ specificity and sensitivity.

The presented case showed: typical histopathology findings, pathergy, erythematous periphery of the ulcer, typical anterior shin localization and therapeutic effectiveness of the immunosuppressive treatment.

Pathergy is an important sign supporting the diagnosis of PG $(12,13)$. It is defined as development of skin lesions that resist healing after minor tissue trauma as in our case. The lesion classically begins as a pustule, vesicle, or nodule that rapidly progresses into a painful ulcer or erosion with raised borders $(14,15)$. That type of lesions can heal spontaneously or coincidentally with antibiotic treatment. Often patients believe they have been bitten by a spider or other type of insect (16). Early aggressive tissue debridement can worsen the therapy response due to pathergy phenomenon.

The clinical course of PG is not predictable, therefore the treatment should be individualized (17). Differential diagnosis of PG includes infectious (atypical mycobacterial ulcers, cutaneous tuberculosis, cutaneous leishmaniasis, sporotrichosis, ecthyma gangrenosum, syphilis) and noninfectious diseases (vasculitis, cutaneous malignancies, drug-induced conditions) (18). Skin biopsy and microbiology tests are crucial for the diagnosis. In the presented case differential diagnosis with ecthyma gangrenosum is important, because of the presence of Pseudomonas aeruginosa in the microbial culture test. The typical massive neutrophilic infiltrate confirms the diagnosis of PG (in contrast, the histopathology of ecthyma gangrenosum shows vascular necrosis with few inflammatory cells). We would interpret the presence of Pseudomonas aeruginosa as contamination of a preexisting PG lesion, which is common for cases of ulcers with prolonged healing.

The treatment of PG remains challenging. Thorough physical examination and comorbidity investigation are crucial for the precise diagnosis and treatment of PG. For mild cases high-potency topical steroids, intralesional application of triamcinolone acetonide and calcineurin inhibitors are mostly used with good therapeutic response $(19,20)$. For severe $P G$, first-line therapy includes systemic steroids or cyclosporine (21). Second-line options are: methotrexate, azathioprine, mycophenolate mofetil, cyclophosphamide, dapsone, thalidomide, colchicine and intravenous immunoglobulins used to decrease the steroid dose in maintenance therapy or in combination with first-line agents for recalcitrant PG (21). Recently, biological agents are also used for treatment of PG $(21,22)$. TNF- $\alpha$ inhibitors prescribed for inflammatory bowel diseases (Crohn's disease and ulcerative colitis) can also improve PG associated with these conditions (23). IL-1 is the key inflammatory mediator triggering release of chemokines involved in neutrophil recruitment and activation. Anakinra and canakinumab are both therapies that block IL-1 and have been used to treat refractory PG (24). New clinical trials show that biologic agents could be an alternative for treatment of severe and refractory PG cases $(25,26)$.

\section{REFERENCES}

1. Pereira N, Brites MM, Gonçalo M, Tellechea $\mathrm{O}$, Figueiredo A. Pyoderma gangrenosum - a review of 24 cases observed over 10 years. Int J Dermatol. 2013; 52(8):938-45. doi: 10.1111/j.1365-4632.2011.05451.x.

2. Alavi A, Sajic D, Cerci FB, Ghazarian D, Rosenbach M, Jorizzo J. Neutrophilic dermatoses: an update. Am J Clinical Dermatol. 2014; 15(5):413-23. doi: 10.1007/s40257-014-0092-6.

3. Barańska-Rybak W, Kakol M, Naesstromet M, Komorowska O, Sokołowska-Wojdyło M, Roszkiewicz J. A retrospective study of 12 cases of pyoderma gangrenosum: why we should avoid surgical intervention and what therapy to apply. Am Surgeon. 2011; 77(12):1644-9.

4. Tolkachjov SN, Fahy AS, Cerci FB, Wetter DA, Cha SS, Camilleri MJ. Postoperative pyoderma gangrenosum: a clinical review of published cases. Mayo Clin Proc. 2016; 91(9):1267-79.

5. Braswell SF, Kostopoulos TC, Ortega-Loayza AG. Pathophysiology of pyoderma gangrenosum (PG): an updated review. J Am Acad Dermatol. 2015; 73(4):691-8.

6. Su WP, Davis MD, Weenig RH, Powell FC, Perry HO. Pyoderma gangrenosum, clinicoplathologic correlation and proposed diagnostic cri- 
Todor Yordanov, Jenya Dimitrova, Ivanka Temelkova et al.

teria. Int J Dermatol. 2004; 43(11):790-800. doi: 10.1111/j.1365-4632.2004.02128.x.

7. Ahronowitz I, Harp J, Shinkai K. Etiology and management of pyoderma gangrenosum : a comprehensive review. Am J Clin Dermatol. 2012; 13(3):191-211. doi: 10.2165/11595240-000000000-00000.

8. Dinarello CA, Simon A, van der Meer JWM. Treating inflammation by blocking interleukin-1 in a broad spectrum of diseases. Nat Rev Drug Discov. 2012; 11(8):633-52.

9. Bakelants E, van der Hilst J, Corluy L, Achten R, Gyssens I, Messiaen P. The diagnostic tangle of pyoderma gangrenosum: a case report and review of the literature. Netherlands J Med. 2014; 72(10):541-4.

10. Powell FC, Su WP, Perry HO. Pyoderma gangrenosum: classification and management. J Am Acad Dermatol. 1996; 34(3):395-409. doi: 10.1016/ s0190-9622(96)90428-4.

11. 11. Maverakis E, Ma C, Shinkai K, Fiorentino D, Callen JP, Wollina U, et al. Diagnostic criteria of ulcerative pyoderma gangrenosum: a delphi consensus of international experts. JAMA Dermatol. 2018; 154(4):461-6. doi: 10.1001/jamadermatol.2017.5980.

12. Al Ghazal $P$, Herberger $K$, Schaller J, Strölin A, Hoff NP, Goerge T, et al. Associated factors and comorbidities in patients with pyoderma gangrenosum in Germany: a retrospective multicentric analysis in 259 patients. Orphanet J Rare Dis. 2013; 8:136. doi: 10.1186/1750-1172-8-136.

13. Garcovich S, De Simone C, Bertiet E, Marzano AV. Drug management of neutrophilic dermatoses. Expert Rev Clin Pharmacol. 2017;10(10):1119-28. doi: 10.1080/17512433.2017.1356719.

14. Weenig RH, Davis MD, Dahl PR, Su WP. Skin ulcers misdiagnosed as Pyoderma gangrenosum. New Eng J Med. 2002; 347(18):1412-8.

15. Callen JP, Jackson JM. Pyoderma gangrenosum: an update. Rheumatic Dis Clin North America. 2007; 33(4):787-802. doi: 10.1016/j.rdc.2007.07.016.

16. Yang S, Kampp J. Common dermatologic procedures. Med Clin North America. 2015; 99(6):130521. doi: 10.1016/j.mcna.2015.07.004.

17. Ruocco E, Sangiuliano S, Gravina AG, Miranda A, Nicoletti G. Pyoderma gangrenosum: an updated review. J Eur Acad Dermatol Venereol. 2009; 23(9):1008-17. doi: 10.1111/j.1468-3083.2009.03199.x.
18. Thomas KS, Ormerod AD, Craig FE, Greenlaw N, Norrie J, Mitchell E, et al. Clinical outcomes and response of patients applying topical therapy for pyoderma gangrenosum: a prospective cohort study. J Am Acad Dermatol. 2016; 75(5):940-9. doi: 10.1016/j.jaad.2016.06.016.

19. Patel F, Fitzmaurice S, Duong C, He Y, Fergus J, Raychaudhuri SP, et al. Effective strategies for the management of pyoderma gangrenosum: a comprehensive review. Acta Derm Venereol. 2015; 95(5):525-31. doi: 10.2340/00015555-2008.

20. Ormerod AD, Thomas KS, Craig FE, Mitchell E, Greenlaw N, Norrie J, et al. Comparison of the two most commonly used treatments for pyoderma gangrenosum: results of the STOP GAP randomised controlled trial. Br Med J. 2015; 350:h2958. doi: 10.1136/bmj.h2958.

21. Feldman SR, Lacy FA, Huang WW. The safety of treatments used in pyoderma gangrenosum. Expert Opin Drug Saf. 2018;17(1):55-61. doi: 10.1080/14740338.2018.1396316.

22. Marzano AV, Fanoni D, Antiga E, Quaglino P, Caproni $\mathrm{M}$, Crosti $\mathrm{C}$, et al. Expression of cytokines, chemokines and other effector molecules in two prototypic autoinflammatory skin diseases, pyoderma gangrenosum and Sweet's syndrome. Clin Exp Immunol. 2014;178(1):48-56. doi: 10.1111/ cei.12394.

23. Arivarasan K, Bhardwaj V, Sud S, Sachdeva S, Puri AS. Biologics for the treatment of pyoderma gangrenosum in ulcerative colitis. Intes Res. 2016;14(4):365-8. doi: 10.5217/ir.2016.14.4.365.

24. Kolios AG, Maul JT, Meier B, Kerl K, Traidl-Hoffmann C, Hertl M, et al. Canakinumab in adults with steroid-refractory pyoderma gangrenosum. Br J Dermatol. 2015; 173(5):1216-23. doi: 10.1111/ bjd.14037.

25. Shavit E, Alavi A, Sibbald RG. Pyoderma gangrenosum: a critical appraisal. Adv Skin Wound Care. 2017; 30(12):534-42. doi: 10.1097/01. ASW.0000526605.34372.9e.

26. Vallini V, Andreini R, Bonadio A. Pyoderma gangrenosum: a current problem as much as an unknown one. Int J Low Extrem Wounds. 2017; 16(3):191-201. doi: 10.1177/1534734617710980. 